\title{
Smac/DIABLO is not released from mitochondria during apoptotic signalling in cells deficient in cytochrome $c$
}

\author{
TM Hansen ${ }^{1,2}$, DJ Smith ${ }^{1,2}$ and P Nagley ${ }^{\star, 1,2}$ \\ 1 Department of Biochemistry and Molecular Biology, Monash University, \\ Clayton, Victoria 3800 , Australia \\ 2 ARC Centre of Excellence in Structural and Functional Microbial Genomics, \\ Monash University, Clayton, Victoria 3800, Australia \\ * Corresponding author: P Nagley, Department of Biochemistry and \\ Molecular Biology, Building 13D, Monash University, Victoria 3800, \\ Australia. \\ Tel: +61-3-9905-3735; Fax +61-3-9905-3726; \\ E-mail: phillip.nagley@med.monash.edu.au
}

Received 17.12.04; revised 19.8.05; accepted 15.9.05; published online 21.10.05 Edited by P Mehlen

\begin{abstract}
We have characterised the apoptotic defects in cells null for cytochrome $c$ (cyt $c-l-$ ). Such cells treated with staurosporine (STS) exhibited translocation to the mitochondria and activation of the proapoptotic signalling molecule Bax but failed to release Smac/DIABLO from these organelles, judged by both confocal microscopy and Western blotting. While reference cells expressing cytochrome $c$ released both it and Smac/DIABLO under a variety of conditions of apoptotic induction, we have never observed release of Smac/DIABLO from cyt $c-l-$ cells. We eliminate the possibility that proteasomal degradation of cytosolically localised Smac/ DIABLO is responsible for our failure to visualise the protein outside the mitochondria. Our findings indicate an unanticipated nexus between release of cytochrome $c$ and Smac/DIABLO from mitochondria, previously thought to be a more or less synchronised event early in apoptosis. We suggest that the failure of cyt $c-I-$ cells to release Smac/DIABLO after recruitment of Bax to mitochondria represents an extreme manifestation of some inherent difference in the regulation of release of these two proteins from mitochondria.

Cell Death and Differentiation (2006) 13, 1181-1190.

doi:10.1038/sj.cdd.4401795; published online 21 October 2005
\end{abstract}

Keywords: mitochondria; cytochrome $c$; Smac/DIABLO; apoptosis

Abbreviations: cyt $c$, cytochrome $c$; IMS, intermembrane space; OM, outer membrane; IAPs, inhibitor of apoptosis proteins; AIF, apoptosis-inducing factor; cyt $c-/-$ cells, cytochrome $c$ knockout mouse embryonic fibroblast cells; STS, staurosporine; MTR, MitoTracker Red; Tdt, terminal deoxynucleotidyl transferase; $\mathrm{CHX}$, cycloheximide

\section{Introduction}

Cytochrome $c$ (cyt $c$ ) plays an important role in both the life and death of animal cells. It is an important component of the mitochondrial respiratory chain responsible for oxidative phosphorylation leading to mitochondrial synthesis of ATP. During apoptosis, cyt $c$ is released from the mitochondrial intermembrane space (IMS) into the cytosol to activate a signalling pathway via formation of the apoptosome (in which Apaf-1 is oligomerised) and activation of caspase-9, and subsequently caspase- 3 , leading to cell death. Permeabilisation of the outer membrane (OM) of the mitochondria, leading to release of cyt $c$ and other apoptogenic proteins from the IMS, is controlled by proteins of the Bcl-2 family. ${ }^{1}$ With many apoptotic stimuli, the proapoptotic Bcl-2 family signalling protein, Bax, is mobilised from the cytosol to the mitochondria where it plays a role in mitochondrial OM permeabilisation. ${ }^{2}$ Among the apoptogenic proteins released from the IMS, aside from cyt $c$, are Smac/DIABLO ${ }^{3,4}$ and apoptosis-inducing factor (AIF). ${ }^{5}$ Once in the cytosol, Smac/DIABLO antagonises the so-called inhibitor of apoptosis proteins (IAPs) that block caspase activation, thus enabling full activation of caspases to occur during apoptotic signalling. For its part in apoptosis, AIF moves to the nucleus, activating degradation of chromosomal DNA.

To further investigate the role of cyt $c$ in apoptosis Li et al. ${ }^{6}$ generated homozygous mice in which the nuclear gene encoding cyt $c$ was rendered nonfunctional (cyt $c-/-$ ). Such homozygous mice died in utero at embryonic day 10.5 , in part due to the deficit in oxidative phosphorylation. Nonetheless, cells from early cyt $c-/-$ embryos were able to be maintained in culture supported by the inclusion of 2-mercaptoethanol, pyruvate and uridine in the growth medium, which compensate for metabolic defects in the absence of cyt $c{ }^{6}$ Significantly, these embryonic fibroblast-like cyt $c-/$ - cells were found to be resistant to apoptosis induced by several agents that act through the mitochondrial pathway, such as staurosporine (STS), UV irradiation and serum starvation. Consistent with the general understanding of apoptotic signalling downstream of mitochondria, in the absence of cyt $c$ in cells, $\mathrm{Li}$ et al. $^{6}$ demonstrated no formation of the apoptosome, no activation of caspase- 9 and no consequent activation of caspase- 3 in cyt $c-/-$ cells.

In order to understand the apoptotic defect in cyt $c-/-$ cells in more detail, it is important to know whether it is only the release of cyt $c$ from the mitochondria and its downstream actions which affect the cell's ability to undergo apoptosis, or whether the absence of cyt $c$ in the knockout cells affects other apoptotic processes at, or downstream of, the mitochondria. Using STS, we have examined if cells lacking cyt cexperience normal apoptotic signalling upstream of the mitochondria, and whether the release of other apoptogenic IMS proteins such as Smac/DIABLO occurs in the absence of cyt $c$. We report 
here that proapoptotic signalling at the mitochondria still occurs in the cyt $c-/-$ cells, as Bax is recruited to the mitochondria in the presence of STS. However, we found that $\mathrm{Smac} / \mathrm{DIABLO}$ is not released from the mitochondria of cyt $c-/-$ cells in response to STS (a protein kinase inhibitor ${ }^{7}$ ) or etoposide (a topoisomerase inhibitor ${ }^{8}$ ). This finding indicates that there are pleiotropic defects in apoptotic signalling effected by mitochondria in cyt $c-/-$ cells and raises the interesting possibility that release of Smac/DIABLO from mitochondria is dependent on either the presence of cyt $c$, as such, or its prior mobilisation from mitochondria to cytosol.

\section{Results}

\section{Cyt $c-l-$ cells mobilise Bax to the mitochondria upon receiving an apoptotic signal}

It has been previously demonstrated that STS engages mitochondria in apoptotic signalling through mobilisation of Bax to these organelles ${ }^{2}$ followed by mitochondrial OM permeabilisation, release of IMS proteins, and subsequent downstream caspase activation. ${ }^{9}$ Therefore, we examined cells by immunocytochemistry with confocal microscopy to see if, in response to STS treatment of cells, Bax distribution changed from its normal, predominantly cytoplasmic, localisation to the mitochondria. Cyt $c-/-$ cells were treated for $24 \mathrm{~h}$ with $250 \mathrm{nM}$ STS and stained with antibodies to Bax, also utilising MitoTracker Red (MTR) to produce a counterstain for mitochondria. Treatment with $250 \mathrm{nM}$ STS for $24 \mathrm{~h}$ has been previously shown in our hands to induce mobilisation of Bax to the mitochondria in 143B human osteosarcoma cells (data not shown). In untreated cells, Bax shows a typical, diffuse cytosolic staining that does not colocalise with MTR (Figure 1a). In contrast, examination of cells treated with STS shows the redistribution of Bax to a punctuate distribution that closely resembles that of MTR (Figure $1 \mathrm{~b}$ ). We also confirmed that the Bax localised at the mitochondria is in an active conformation by immunostaining with the conformation-specific Bax 6A7 antibody. Cyt $c-/-$ cells were treated with STS as previously, but permeabilised with CHAPS buffer, which does not expose the epitope characteristic of activated Bax in untreated cells. Thus, untreated cells show no staining under these conditions (Figure 1c). However, cyt $c-/-$ cells treated with $250 \mathrm{nM}$ STS for $24 \mathrm{~h}$ show staining of activated Bax in a typical mitochondrial location (Figure 1d). STStreated cyt $c-/-$ cells also show morphological changes typical of apoptosis, namely shrinkage and rounding of the cells. ${ }^{1}$ We interpret these data to infer that the upstream pathway of signalling to the mitochondria is intact in the cyt $c-/-$ cells, as treatment with STS causes recruitment of Bax to the mitochondria; moreover in Figure $1 \mathrm{~b}$, the mitochondria themselves show pyknotic morphology in perinuclear aggregates, indicative of an apoptotic response. ${ }^{1}$

\section{Cyt $c-I$ - cells neither degrade DNA nor activate caspase-3 in response to mitochondrial apoptotic signalling}

In order to confirm that apoptotic events downstream of the mitochondria were blocked in the cyt $c-/-$ cells, we
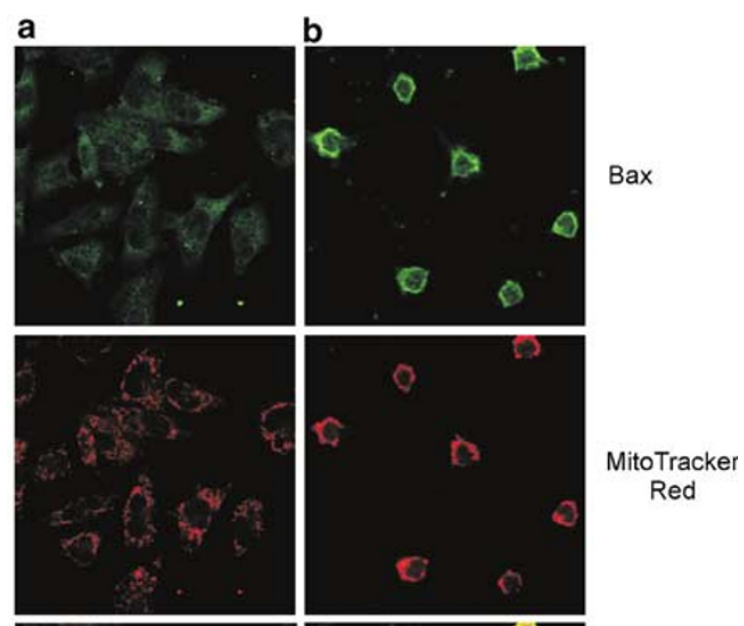

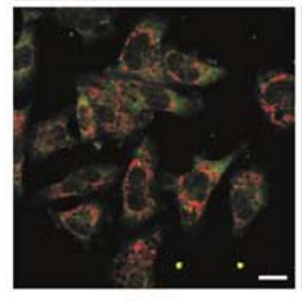

-STS
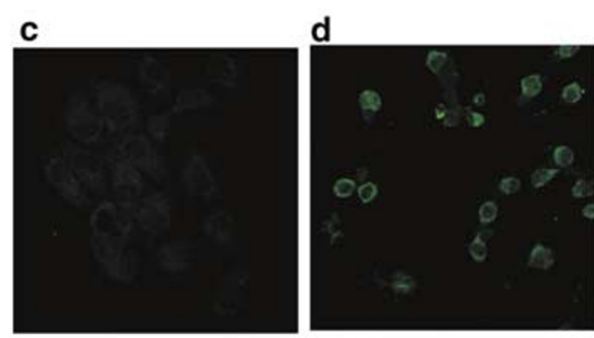

Bax 6A7 antibody
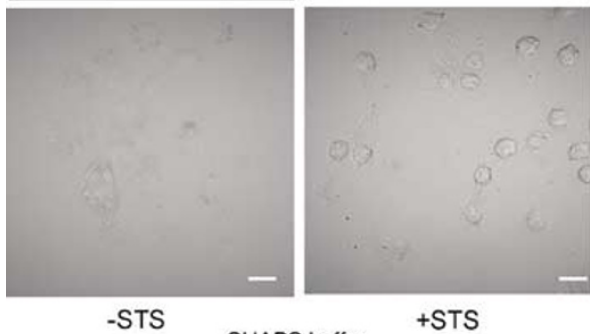

CHAPS buffer

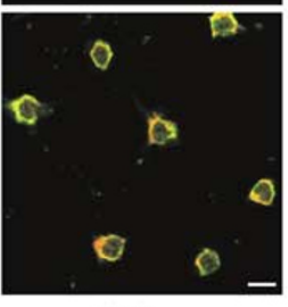

Overlay

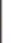$$
\text { (n) }
$$

Figure $1 \mathrm{Bax}$ is mobilised to the mitochondria and assumes an active conformation after STS treatment of cyt $c-/-$ cells. Cyt $c-/-$ cells, (a) either untreated (-STS) or (b) treated with $250 \mathrm{nM} \mathrm{STS} \mathrm{for} 24 \mathrm{~h}$ (+ STS) were fixed, permeabilised with Triton X-100 and subjected to immunocytochemistry using antibodies specific for Bax (with secondary antibody labelled with Alexa 488; top row), having been previously stained with MTR (middle row). Overlays of the confocal microscope images of Bax immunostaining and MTR fluorescence are shown (bottom row). Alternatively, cyt $c-/-$ cells, either untreated (c) or treated with STS as above (d), were permeabilised with CHAPS buffer and subjected to immunocytochemistry under conditions whereby only activated Bax is detected by the conformation-specific Bax antibody (top row). Differential interference contrast (DIC) images are shown (bottom row). Scale bars, $50 \mu \mathrm{m}$

investigated whether chromosomal DNA degradation and caspase activation occurred in these cells after STS treatment. Such DNA degradation was assessed using the 
TUNEL assay, monitored by flow cytometry, to determine the extent of uptake of fluorescently tagged DNA strand termini. Cyt $c-/-$ cells or two reference cell lines (143B and NIH3T3), all when untreated, show very weak fluorescence in the M1 region, indicating little or no DNA degradation (see flow cytometry graphical data in Figure 2a, quantified in Figure $2 b$ ). When cells were treated with $250 \mathrm{nM}$ STS for $24 \mathrm{~h}$, an increase in fluorescence due to DNA degradation is seen by movement of the fluorescence peak to the right in data from the 143B cells (human osteosarcoma), and to a lesser extent in the NIH3T3 cells (embryonic mouse cells with a normal level of cyt $c$ ) (Figure 2a). However, the cyt $c-/-$ cells show no shift in the peak fluorescence in flow cytometry (Figure 2a), with no significant change in the quantified extent of terminal deoxynucleotidyl transferase (Tdt) binding between treated and untreated samples (Figure $2 \mathrm{~b}$ ), indicating that no DNA degradation has occurred in response to STS treatment. To detect apoptotic changes in one of the reference cell lines used, the mouse embryonic fibroblast NIH3T3 cells, and to determine that this lack of response in the STS-treated cyt $c-/-$ cells is not due to the strength of the apoptotic stimulus, NIH3T3 and cyt $c-/-$ cells were treated with $2 \mu \mathrm{M}$ STS and examined for DNA degradation. Quantified data (Figure 2b) show a large increase in DNA degradation in NIH3T3 cells treated with the stronger stimulus, with only a small amount of degradation appearing in the cyt $c-/-$ cells. We postulate that this slight increase in degradation is due to the damage that occurs because of necrosis rather than apoptosis.

Like DNA degradation, caspase-3 activation normally occurs in response to apoptotic signalling through the mitochondrial pathway after formation of the apoptosome and activation of caspase-9. We tested if caspase-3 was being activated in the cyt $c-/-$ cells. Cleavage of the caspase-3-specific peptide substrate, DEVD-AFC to generate a fluorescent product, was measured to determine the level of caspase-3 activation. Fluorescent values obtained from cell lysates were standardised to a percentage of maximum fluorescence, arbitrarily taken to be that of the 143B cells treated for $24 \mathrm{~h}$ with $250 \mathrm{nM}$ STS. Whereas the 143B cells, and to a lesser extent the NIH3T3 cells, showed significant activation of caspase- 3 after $24 \mathrm{~h}$ STS treatment, there was no such activation of caspase- 3 in cyt $c-/-$ cells likewise treated. NIH3T3 and cyt $c-/-$ cells were also treated with $2 \mu \mathrm{M}$ STS to determine if a stronger apoptotic stimulus could
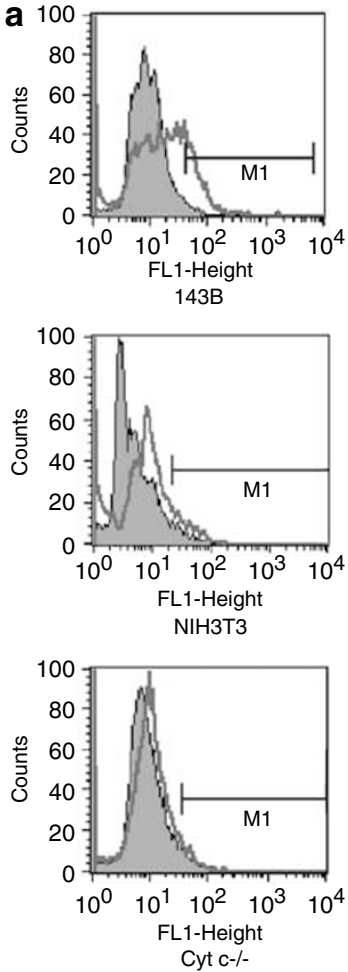

b

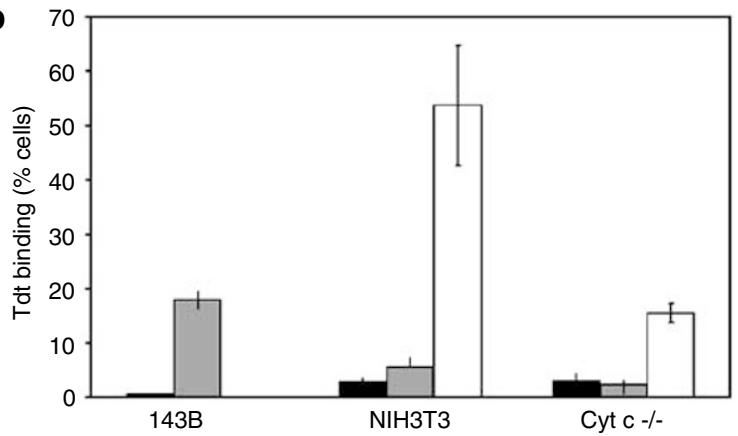

C

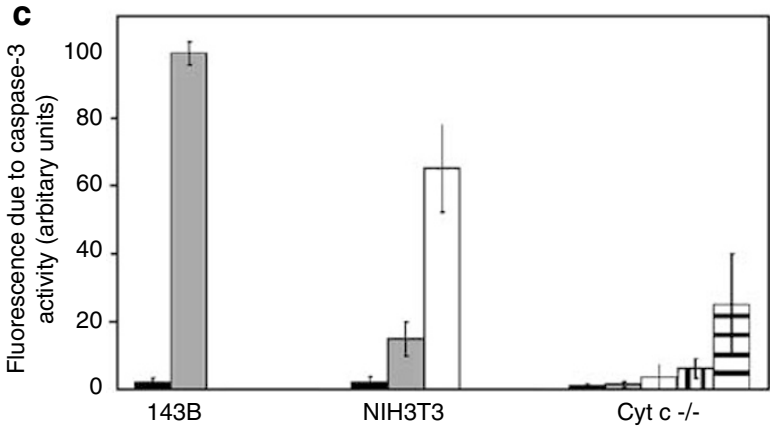

Figure 2 Impaired downstream apoptotic markers in cyt $c-/$ - cells after treatment with STS. DNA degradation was analysed by flow cytometry using TUNEL labelling. (a) Graphical flow cytometric data from reference cyt c-positive cells (143B and NIH3T3) and cyt $c-1-$ cells. Curves with solid or clear areas show data on untreated cells or cells treated with $250 \mathrm{nM}$ STS for $24 \mathrm{~h}$, respectively. M1 indicates the region of TUNEL-positive cells, that is, with a significant extent of binding of the fluorescent antibody specific for terminal deoxynucleotidyl transferase (Tdt). (b) Quantified data of DNA degradation after $24 \mathrm{~h}$ treatment with $250 \mathrm{nM}$ STS. Bars indicate the percentage of cells with increased fluorescence due to Tdt binding, that is, in M1 region of graphical data from flow cytometry, examples of which are shown in (a). Black bars, untreated cells; grey bars, cells treated with $250 \mathrm{nM} \mathrm{STS}$ for $24 \mathrm{~h}$; white bars, cells treated with $2 \mu \mathrm{M}$ STS for $24 \mathrm{~h}$. Results shown are from three independent experiments; error bars indicate standard deviation. (c) Caspase-3 activation in the cells indicated was analysed by measuring cleavage of DEVD-AFC, which generates a fluorescent product. Fluorescence values were standardised to a percentage of maximum, arbitrarily taken to be that of the positive control (set at 100 units), namely $143 \mathrm{~B}$ cells treated for $24 \mathrm{~h}$ with $250 \mathrm{nM}$ STS. Black bars, untreated cells; grey bars, cells treated for with $250 \mathrm{nM} \mathrm{STS}$ for $24 \mathrm{~h}$; white bars, cells treated with $2 \mu \mathrm{M}$ STS for $24 \mathrm{~h}$; vertical stripes, $5 \mathrm{ng} / \mathrm{ml}$ TNF- $\alpha$; horizontal stripes, $2 \mathrm{ng} / \mathrm{ml} \mathrm{TNF}-\alpha$ and $1 \mu \mathrm{g} / \mathrm{ml} \mathrm{CHX.} \mathrm{Results} \mathrm{are} \mathrm{from} \mathrm{three} \mathrm{independent} \mathrm{experiments;} \mathrm{error} \mathrm{bars}$ indicate standard deviation 
induce caspase-3 activation and apoptosis. However, while a much higher level of caspase-3 activation was observed in NIH3T3 cells treated with $2 \mu \mathrm{M}$ STS compared to $250 \mathrm{nM}$ STS, no increase was evident in the $2 \mu \mathrm{M}$ STS treated cyt $c-/-$ cells (Figure $2 c$ ), similar to the results obtained for DNA degradation (Figure $2 b$ ).

To confirm that the cyt $c-/-$ cells are indeed able to activate caspase-3 under some conditions, we also treated cyt $c-/-$ cells with TNF- $\alpha$ with and without cycloheximide (CHX). The combination of these reagents induces apoptosis via the death receptor pathway. As shown in Figure $2 c$, we observe that cyt $c-/-$ cells are able to activate caspase- 3 in response to TNF- $\alpha$ in the presence of $\mathrm{CHX}$ in confirmation of the earlier findings of $\mathrm{Li}$ et al. ${ }^{6}$ We thus conclude that the apoptotic signalling pathway downstream of the mitochondria is not activated in the cyt $c-/-$ cells, and that delivering a stronger apoptotic stimulus is insufficient to overcome the block in the apoptotic pathway effected at the mitochondrial level.

\section{Cyt $c-I-$ cells do not release Smac/DIABLO from mitochondria upon apoptotic stimuli}

We asked if the lack of caspase activation in cyt $c-/-$ cells subsequent to apoptotic stimuli can be explained solely on the failure to release of cyt $c$ from mitochondria, or whether deficiencies in release of other apoptogenic mitochondrial proteins contributes to the deficits in downstream pathways of apoptosis in these cells. Accordingly, cells treated with $250 \mathrm{nM}$ STS were examined by immunocytochemistry to monitor the release of both Smac/DIABLO and cyt $c$ from mitochondria. Release of Smac/DIABLO (and cyt $c$ ) is seen in reference cell lines after only $8 \mathrm{~h}$ of STS treatment (Figure 3a). Quantification of the population of treated cells shows that $15-20 \%$ of cells from the reference cells lines have released Smac/DIABLO after $8 \mathrm{~h}$ of STS treatment, a similar proportion to those releasing cyt $c$ at this time (Figure $3 b$ ). No significant release of Smac/DIABLO could be observed in cyt $c-/-$ cells treated with STS even for the extended period of $24 \mathrm{~h}$ (Figure 3a). Concordantly, with quantification, there is no increase in the percentage of cyt $c-l-$ cells that have released Smac/DIABLO after STS treatment (Figure 3b).

To confirm and extend the data obtained using immunocytochemistry, Western blotting was performed on fractionated cells treated with STS for periods up to $24 \mathrm{~h}$. Under these conditions, reference 143B cells show clear release of Smac/DIABLO and cyt $c$ from mitochondria, while NIH3T3 cells show a smaller, but still significant, release of both proteins (Figure $4 a$ ). In contrast, the cyt $c-/-$ cells do not show detectable translocation of Smac/DIABLO into the cytosolic fraction under the same conditions.

To verify that release of Smac/DIABLO from mitochondria of cyt $c-1-$ cells was not merely delayed compared to that of our reference cell lines, we extended the STS treatment ( $250 \mathrm{nM})$ over periods of up to 7 days (168h, Figure 4c). This extended STS treatment did not lead to detectable release of Smac/DIABLO from mitochondria of cyt $c-/-$ cells (Figure 4c), in contrast to reference cell lines, which show release of Smac/DIABLO as early as $8 \mathrm{~h}$ with STS treatment (Figure 4a). Even though we observed no caspase-3
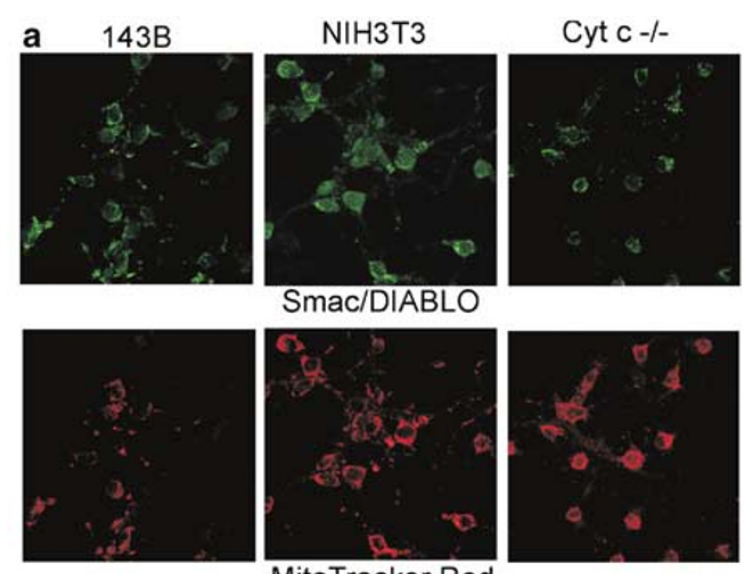

Smac/DIABLO
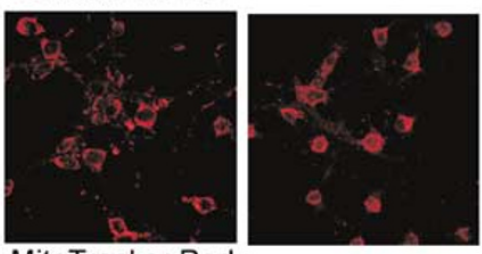

MitoTracker Red
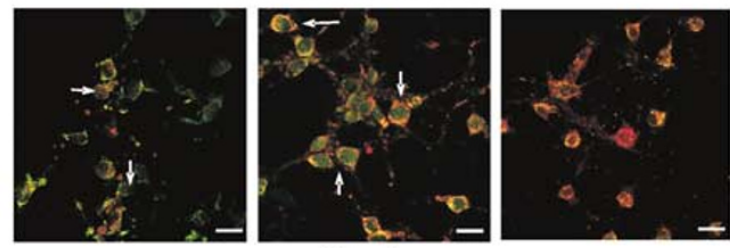

Overlay

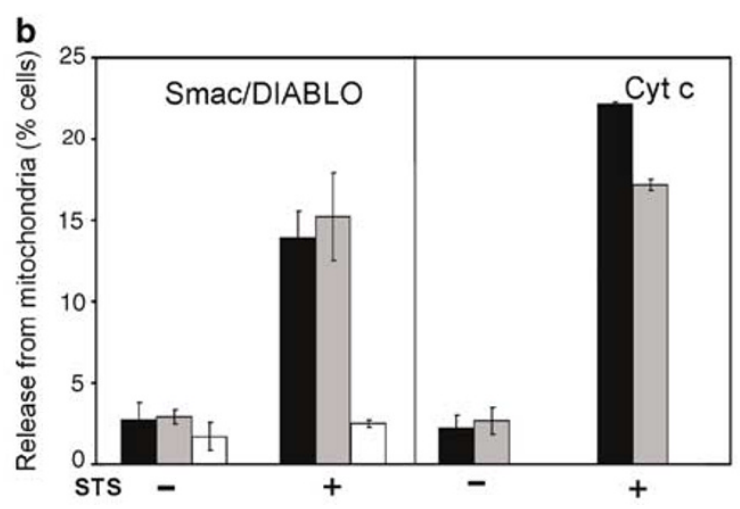

Figure 3 Confocal microscope analysis of release of Smac/DIABLO and cyt $c$ from mitochondria of cells treated with STS. Representative images of cells subjected to immunocytochemistry using antibodies specific for Smac/DIABLO (secondary antibody labelled with Alexa 488; top row), having been previously stained with MTR (middle row). Overlays of the Smac/DIABLO immunostaining and MTR fluorescence are shown (bottom row). Scale bars $50 \mu \mathrm{m}$. (a) Reference cells $143 \mathrm{~B}$ and NIH3T3 were treated with $250 \mathrm{nM}$ STS for $8 \mathrm{~h}$ and cyt $c-/-$ cells likewise treated for $24 \mathrm{~h}$. Arrows in overlays (bottom row) indicate cells that have released Smac/DIABLO from the mitochondria. (b) Quantification of cells releasing Smac/DIABLO or cyt $c$ from mitochondria in untreated cells $(-)$ or after treatment of cells with STS $(+)$. Black bars, 143B; grey bars, NIH3T3; white bars, cyt $c-1-$ cells. Results are calculated from two independent experiments with a minimum of 150 cells counted per experiment; error bars indicate standard deviation. Note that in (a) two cells in the rightmost series of images show weak staining for Smac/DIABLO relative to that for MTR. This is not an effect of STS treatment because even in untreated cells a similar proportion of cyt $c-/-$ cells fail to stain efficiently with antibodies such as those used for Smac/DIABLO here (data not shown)

activation and limited DNA degradation in cyt $c-/-$ cells when treated with an increased concentration of STS, it has been reported previously that under some conditions Smac/ DIABLO is only released from mitochondria with much stronger apoptotic stimuli than those required to release cyt $c^{10}{ }^{10}$ Therefore, an increased concentration of STS $(2 \mu \mathrm{M})$, as 
a

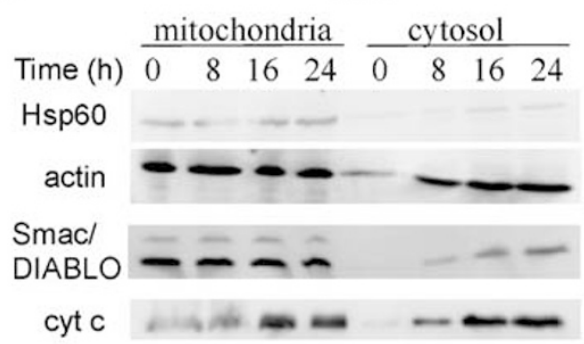

b

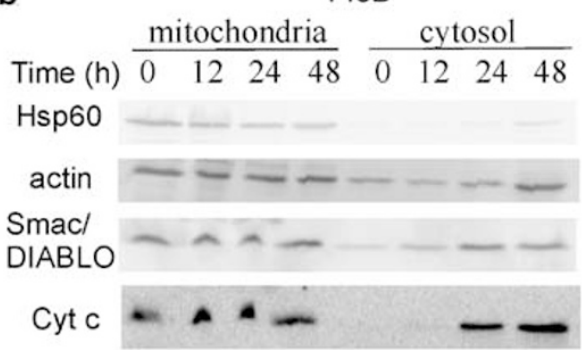

c

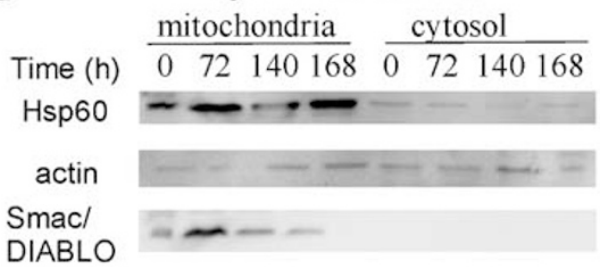

NIH3T3

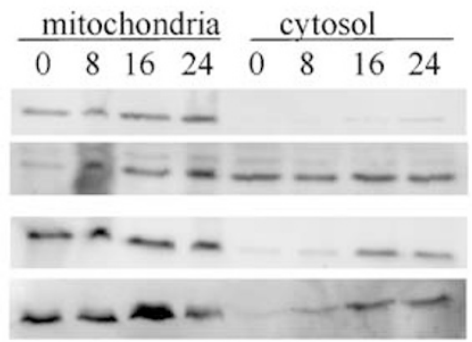

STS treatment

$\mathrm{NIH} 3 \mathrm{~T} 3$

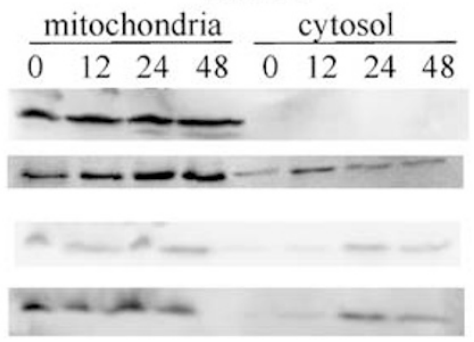

Etoposide treatment

d Cyt c - $-2 \mu \mathrm{M} \mathrm{STS}$

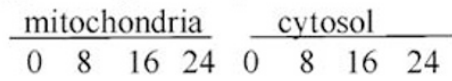

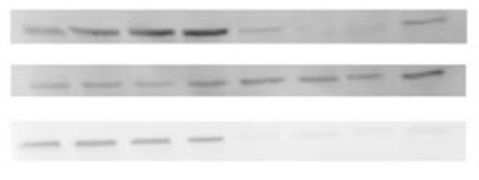

Cyt C -/-
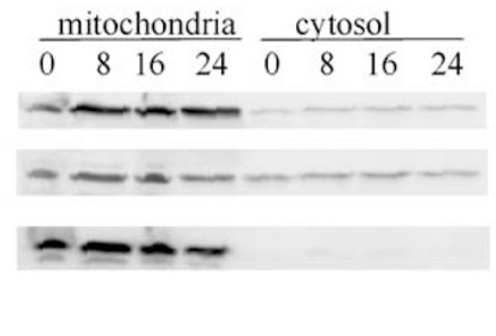

Cyt c - -

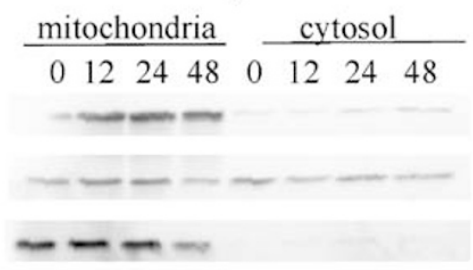

e $\rho^{0}$ cells $250 \mathrm{nM} \mathrm{STS}$

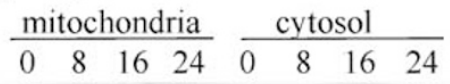

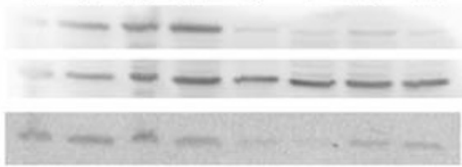

Figure 4 Western blot analysis of release of Smac/DIABLO and cyt c from mitochondria. Cells subjected to treatments for the times indicated were permeabilised with digitonin and fractionated into mitochondrial and cytosolic fractions. Samples of both fractions were subjected to SDS-PAGE and Western blotting to detect Smac/ DIABLO and cyt $c$ (with reference proteins Hsp60 and actin also detected). Treatment regimes were as follows: (a) $250 \mathrm{nM}$ STS; (b) $50 \mu \mathrm{M}$ etoposide; (c) $250 \mathrm{nM}$ STS over a 7-day period; (d) $2 \mu \mathrm{M}$ STS; (e) $\rho^{0} 206$ cells treated with $250 \mathrm{nM}$ STS. Other indications, including cell line and duration of treatment, are shown in the relevant panels

used previously, was used to treat the cyt $c-/-$ cells. Significantly, release of Smac/DIABLO from mitochondria was not seen even after $24 \mathrm{~h}$ treatment at the higher STS concentration (Figure 4d), compared to release of Smac/ DIABLO from mitochondria of another mouse embryonic fibroblast cell line, NIH3T3, observed at $8 \mathrm{~h}$ after treatment with $2 \mu \mathrm{M} \mathrm{STS}$ (data not shown). This implies that the cyt $c-/-$ cells are resistant to apoptotic induction via the mitochondrial pathway, and that increasing the level of stimulus is insufficient to overcome this resistance.

To test whether this distinction between cyt $c$-positive cells and those lacking cyt $c$ occurred with other apoptotic stimuli, cells were treated with etoposide, a topoisomerase inhibitor that causes DNA-damage induced apoptosis via cyt $c$ release and caspase activation. ${ }^{11}$ Reference cell lines 143B and NIH3T3 treated with $50 \mu \mathrm{M}$ etoposide release both cyt $c$ and Smac/DIABLO from the mitochondria as early as $12 \mathrm{~h}$ after treatment (Figure 4b). In contrast, the cyt $c-/-$ cells show no detectable release of Smac/DIABLO into the cytosolic fraction even after $48 \mathrm{~h}$ treatment with etoposide. This strengthens the observation that apoptotic inducers acting through the mitochondrial pathway are unable to induce Smac/DIABLO release from the mitochondria in cyt $c-/-$ cells, and that this phenomenon is not unique to STS as an inducer.

It may be proposed that an explanation for the failure of Smac/DIABLO release in the cyt $c-/-$ cells is due to the function of cyt $c$ in the respiratory chain and not its role in apoptosis. However, $\rho^{0} 206$ cells from 143B TK- human osteosarcoma cells which are also respiration-deficient, show release of cyt $c^{12}$ and Smac/DIABLO (Figure 4e) from mitochondria after STS treatment. This indicates that a deficiency in respiration is not sufficient in itself to retard Smac/DIABLO release.

\section{The failure to observe cytosolically localised Smac/DIABLO in cyt $c-l-$ cells is not due to proteasome activity}

Evidence has been presented that under some circumstances Smac/DIABLO is susceptible to ubiquitinylation and subsequent degradation by the proteasome, after release from the 
mitochondria. ${ }^{13}$ To exclude the possibility that this may be the reason that we observe no increase of Smac/DIABLO in the cytosolic fraction of cyt $c-/-$ cells after STS treatment (Figure $4 a$ ), cyt $c-1-$ cells were treated with the proteasome inhibitor MG-132 prior to STS treatment. Initially we needed to determine a concentration of MG-132 sufficient to inhibit the proteasome in the cyt $c-/-$ cells. As shown in Figure $5 \mathrm{a}$, the minimum concentration found to inhibit the proteasome in the cyt $c-/-$ cells was $1 \mu \mathrm{M}$, judged by the accumulation of ubiquinitinylated proteins. However, treating cyt $c-/-$ cells with $1 \mu \mathrm{M}$ MG-132 resulted in detectable Smac/DIABLO release in to the cytosol, even when no STS was added (Figure 5b). However, since the mitochondrial matrix protein Hsp60 is also observed to be released into the cytosol with MG-132 treatment, we infer that some non-specific permeabilisation of mitochondria has occurred to a limited extent in cyt $c-/-$ cells. Note that Smac/DIABLO and Hsp60 are released in parallel at MG-132 concentrations at or above the threshold of $1 \mu \mathrm{M}$ at which the proteasome is inhibited (Figure 5b). Significantly, when cyt $c-/-$ cells were pretreated with $1 \mu \mathrm{M} \mathrm{MG}-132$ and then subjected to STS treatment, there was no increase in the amount of cytosolically localised Smac/ DIABLO (Figure $5 \mathrm{c}$ ). The intensity of the band was again in

a

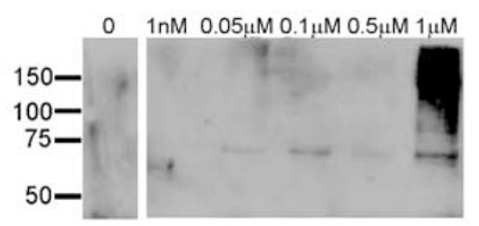

b
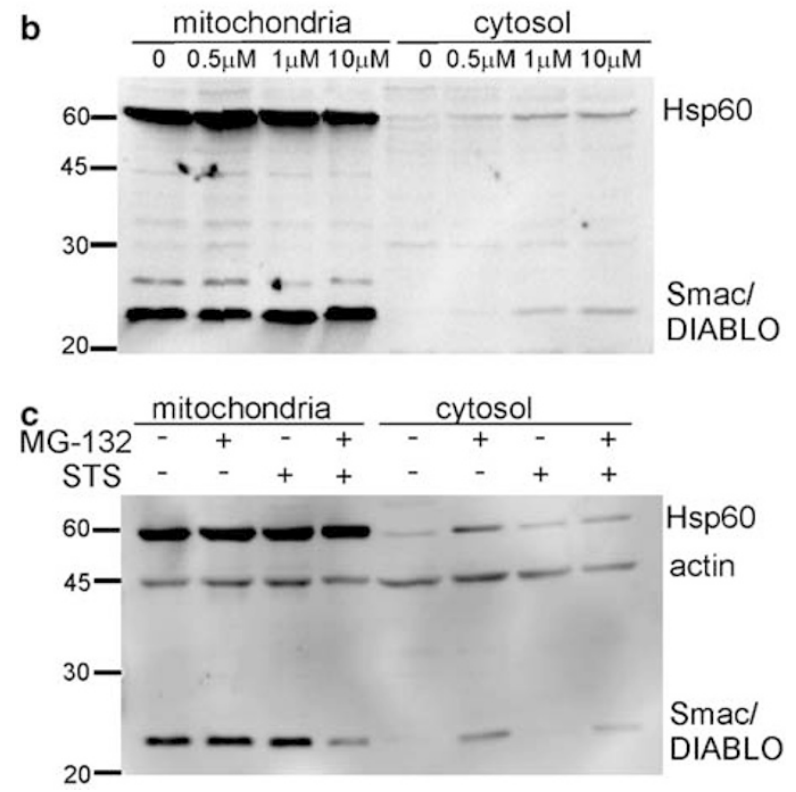

Figure 5 Treatment of cyt $c-/-$ cells with the proteasome inhibitor MG- 132. (a) Cells treated with various concentrations of MG-132 as indicated; whole-cell lysates were probed with antibodies to ubiquitin. (b) Cells were treated with various concentrations of MG-132, then permeabilised with digitonin, separated into mitochondrial and cytosolic fractions and probed for release of Smac/ DIABLO. (c) Cells were treated with $1 \mu \mathrm{M} \mathrm{MG}-132$ prior to treatment with $250 \mathrm{nM}$ STS, fractionated and probed for release of Smac/DIABLO. Identical results to those shown in (c) have been obtained on three separate occasions parallel with that of the Hsp60 band under these conditions. We therefore conclude that Smac/DIABLO is not released into the cytosol of cyt $c-/-$ cells during STS treatment, under conditions where MG-132 pretreatment blocks proteasome activity.

\section{Discussion}

The mitochondria play a key role in apoptosis elicited by many stimuli. These signals are normally regulated at the mitochondria by the Bcl-2 family of proteins, containing both proapoptotic and antiapoptotic members, such as Bax or Bcl-2 itself, respectively. Here we have shown defects in the mitochondrial apoptotic signalling pathway of cyt $c-/-$ cells, which extends beyond the possibility that downstream signalling is blocked simply due to the absence of cyt $c$. Notwithstanding the mobilisation of Bax to the mitochondria in the cells null for cyt $c$ after STS treatment, these mitochondria are evidently unable to release Smac/DIABLO. These results raise interesting questions about the possible hierarchical order of release of IMS proteins and the role cyt $c$ itself may play in outer mitochondrial membrane permeabilisation leading to release of other IMS proteins. Moreover, the apoptotic effects in cyt $c-1-$ cells extend beyond failure to activate the apoptosome; the consequential inability to release Smac/ DIABLO from mitochondria would prevent inhibition of IAPs that would otherwise enable maximal caspase activation.

It would appear that the apoptotic stimuli provided are capable of inducing the appropriate signalling to the mitochondria in the cyt $c-/-$ cells. Thus, with STS treatment, Bax is mobilised to the mitochondria from its prior location in the cytoplasm, and then activated. Bax would normally be expected to overcome antiapoptotic inhibition by proteins such as Bcl-2 and $\mathrm{Bcl}-\mathrm{x}_{\mathrm{L}}$ and be involved in permeabilisation of the outer membrane leading to release of cyt $c$ and other IMS proteins. In the cyt $c-/-$ cells, it appears that while the proapoptotic Bax signal is received, it does not in this case generate release of Smac/DIABLO from mitochondria. While we have determined that $B a x$ is indeed in its active conformation at the mitochondria, we have not directly investigated if oligomerisation of Bax occurs. If the cyt $c$ deficiency is preventing Bax oligomer formation, it may also be preventing the formation of Bax channels at the mitochondrial membrane. While these channels may not be the mechanism, as such, for release of cyt $c$ or Smac/DIABLO, formation of Bax oligomers can induce cyt $c$ release in isolated mitochondria, ${ }^{14}$ and Bax oligomerisation induced by tBid in outer membrane vesicles is able to induce the formation of lipidic pores which are able to release molecules up to $2000 \mathrm{kDa}^{15}$

From previous studies, using Western blotting and confocal microscopy, it has been inferred that Smac/DIABLO and cyt $c$ are both released into the cytosol more or less at the same time after apoptotic treatments. $3,4,16,17$ Thus, release of Smac/DIABLO and cyt $c$ is generally viewed to occur at the same time, via the same pathway. Studies on Smac/DIABLO release using Smac-YFP fusion proteins show its release to have a similar onset time as cyt $c$ release, but to take up to three times as long to be released compared to cyt $c .^{16,17}$ The 
longer kinetics of release are proposed to be due to the larger size of monomeric Smac/DIABLO (23 kDa) compared to that of cyt $c(14 \mathrm{kDa})$ and the existence of Smac/DIABLO as an arch-shaped homodimer, ${ }^{18}$ which also increases its size relative to that of cyt $c$. However, the extended duration of Smac/DIABLO release might also be influenced by the use of fluorescent protein tags. Recent work on STS-treated 143B cells using immunocytochemistry to monitor the kinetics of release of cyt $c$ and Smac/DIABLO with respect to one another has revealed a substantial portion of cells over several hours of STS treatment, in which cyt $c$ release has occurred but not that of Smac/DIABLO (MLR Lim and $P$ Nagley, unpublished data). Thus, not only is Smac/DIABLO slower to exit the mitochondria once release has been initiated, but the initiation of release of Smac/DIABLO may be significantly delayed with respect to that of cyt $c$ under some circumstances.

In the cyt $c-/-$ cells, we showed that Smac/DIABLO was unable to be released from mitochondria not only under mild conditions that induced release in cyt $c$-positive mouse embryonic fibroblast cells but also under more vigorous conditions of apoptotic stimulation, such as higher concentrations of STS and extended treatment times. Other apoptotic inducers such as etoposide (Figure 4b), HA14-1 (a small molecule $\mathrm{Bcl}-2$ inhibitor ${ }^{19,20}$ ) and serum starvation (data not shown) were also unable to induce Smac/DIABLO release in the cyt $c-/-$ cells, while inducing both Smac/DIABLO and cyt $c$ release in cyt $c$-positive cell lines.

Under some circumstances, Smac/DIABLO has been shown to be degraded by the proteasome once released into the cytosol, ${ }^{13}$ which can be prevented by the proteasome inhibitor MG-132. Treatment with MG-132 itself in the cyt $c-/-$ cells appeared to cause a limited extent of nonspecific permeabilisation of mitochondrial membranes, leading to some release of both Smac/DIABLO and the mitochondrial matrix protein Hsp60 (Figure $5 \mathrm{~b}$ ). The nature of this permeabilisation in cyt $c-/-$ cells has not been investigated further, except to note that the mobility of the Smac/DIABLO band observed corresponds to that of processed protein (after import) rather than precursor, whose size is some $5 \mathrm{kDa}$ larger and easily distinguished by its mobility on SDS-PAGE as applied here (data not shown). It is to be emphasised that the amount of Smac/DIABLO released into the cytosol under these conditions did not increase when cells were treated with STS. Therefore, the possible release or Smac/DIABLO from mitochondria and its rapid degradation by the proteasome in the cytosol of cyt $c-/-$ cells is eliminated as the explanation of why we were unable to observe Smac/DIABLO in the cytosol of such cells after treatment with apoptotic reagents such as STS or etoposide.

The lack of detectable Smac/DIABLO release in the cyt c-/- cells with multiple apoptotic stimuli suggests that Smac/ DIABLO is unable to be released independently of cyt $c$. The question arises as to whether this lack of release is due to cyt $c$ and Smac/DIABLO being released via the same mechanism, but there is a sequential requirement for cyt $c$ release to proceed before that of Smac/DIABLO, or whether the defect is in some signalling circuit involving mitochondria which would normally be initiated by cyt $c$ after its release into the cytosol.
The dependence of Smac/DIABLO release on cyt $c$ could be mechanistic, where cyt $c$ is required either directly or as a cofactor to activate the mechanism of Smac/DIABLO release. This may be 'internal' to mitochondrial reorganisation whereby mobilisation of cyt $c$ from regions deep within the cristae, into the IMS and through the outer membrane, enables or promotes Smac/DIABLO release. tBid has been shown to promote reorganisation of mitochondrial cristae, mobilising cyt $c$ and making it available for release. ${ }^{21}$ This cristal reorganisation may also be required for Smac/DIABLO to be available for release. Resolving whether cyt $c$ and Smac/ DIABLO are released via the same mechanism may address the possibility of mechanistic interdependence; if the release mechanisms are different, this may imply a role for cyt $c$ in assisting pore formation in the OM for release of other IMS proteins.

Alternatively, the dependence on cyt $c$ of Smac/DIABLO release may arise from some signalling mechanism external to mitochondria. Thus, a signal may be initiated by cyt $c$, once released into the cytosol, creating a feed-forward mechanism that enables Smac/DIABLO release. Feed-forward or signal amplification systems for mitochondria-induced apoptosis have been reported, for example, $\mathrm{Ca}^{2+}$ signalling from the $\mathrm{ER}^{22}$ In this feed-forward model, apoptotic stimuli promote small amounts of cyt $c$ to be released, which move to the ER, causing release of $\mathrm{Ca}^{2+}$ into the cytosol. ${ }^{22}$ This released $\mathrm{Ca}^{2+}$ is suggested to activate the mitochondria to cause extensive outer membrane permeabilisation and release of the remaining cyt $c .^{22}$ Release of Smac/DIABLO by this mechanism was not examined in these studies, but if general OM permeabilisation occurs, as is proposed to occur with mitochondrial $\mathrm{Ca}^{2+}$ overload, ${ }^{23}$ release of Smac/DIABLO would certainly be anticipated.

Such a feed-forward circuit is to be distinguished from that involving downstream caspase activation which is reportedly required for release of AIF. ${ }^{24}$ As there is little or no caspase-3 activation in the cyt $c-/-$ cells $^{6}$ (and Figure $2 c$ herein), one would expect release of AIF to be retarded. Indeed, we have not been able to observe detectable AIF release in the cyt $c-/-$ cells after STS treatment (TM Hansen, unpublished data) and AIF release from the mitochondria was not seen in colon cancer cells that have had cyt $c$ expression reduced by RNA $i^{25}$ These results therefore indicate that cyt $c$ plays a role in mediating or signalling the release of other apoptogenic proteins from the IMS during apoptosis.

The cyt $c-/-$ cells undergo morphological changes after STS treatment, such as cell shrinkage and clustering of the mitochondria around the nucleus, which are typical of apoptosis. ${ }^{1}$ Further work has also indicated mitochondrial stress consequential to apoptotic stimuli in cyt $c-/-$ cells, such that mitochondria in the cyt $c-/-$ cells have lost their membrane potential after $24 \mathrm{~h}$ STS treatment as do 143B cells (TM Hansen, unpublished data), characteristic of mitochondria in the later stages of apoptosis, after caspase activation. ${ }^{26}$ Further work is required to elucidate how the cyt $c-/-$ cells normally maintain their membrane potential in the face of impaired respiratory electron transport, what signals are causing the reduction of membrane potential in cyt $c-/-$ cells with STS treatment and how this affects the various components of the cell that are dependent on mitochondrial function. 
In this paper, we have focused on the role of cyt $c$ in apoptosis, rather than in respiratory electron transport, as the potential explanation for why the cyt $c-/-$ cells fail to undergo apoptosis. We do not consider it likely that the respiration deficiency affects the cells' ability to die by apoptosis. This is because 143B $206 \rho^{0}$ cells (lacking mitochondrial DNA), which are absolutely respiration-deficient, are able to release both cyt $c^{27}$ and Smac/DIABLO (Figure 4e) from mitochondria during STS-induced apoptosis. There may, however, be some subtle disjunction of respiratory electron transport in cyt $c-/-$ cells that influences apoptotic signalling.

Although the mouse embryonic cell line NIH3T3 provided a useful comparator in the present studies, cyt $c-/-$ cells in which cyt $c$ expression has been genetically restored would represent an important control in confirming that the observed defects in cyt $c-/-$ cells relate solely to the absence of cyt $c$ and not to some additional defect. However, in contrast to $\mathrm{Li}$ et al., ${ }^{6}$ we have thus far been unable to achieve significant levels of transfection of cyt $c-/-$ cells using vectors designed for expression of cyt $c$, which would permit such experiments to be performed. Nonetheless, Li et al. ${ }^{6}$ showed that on restoration of cyt $c$ expression in the cyt $c-/-$ host cells, the cells underwent apoptosis in response to UV irradiation, indicating there that was no other intrinsic apoptotic signalling defect in these cells. Downstream apoptotic mechanisms are still functional in cyt $c-/-$ cells as shown by treatment with apoptotic inducers acting through the death-receptor pathway. ${ }^{6}$ Concordantly, we have also observed caspase-3 activation in cyt $c-/-$ cells treated with TNF- $\alpha$ and $\mathrm{CHX}$, as for Li et al. ${ }^{6}$ Such activation of caspase-3 is independent of mitochondrial apoptotic signalling and presumably occurs via an enhanced death-receptor pathway. ${ }^{6}$

In conclusion, the failure of cyt $c-/-$ cells to release Smac/ DIABLO, after the recruitment of apoptogenic signals to the mitochondria in the form of Bax, may represent an extreme manifestation of some inherent difference in the process whereby cyt $c$ and Smac are released from the mitochondria. The dependence of Smac/DIABLO release on cyt $c$ may be due to a requirement for cyt $c$ to activate a mechanism for Smac/DIABLO release, or the lack of cyt $c$ abolishes a signalling pathway required for the release of Smac/DIABLO. Elucidation of the molecular regulation of such differential release pathways is a current priority.

\section{Materials and Methods}

\section{Materials and antibodies}

RPMI 1640, DMEM and foetal calf serum were purchased from JRH Biosciences (Lenexa, KS, USA), penicillin/streptomycin from CSL Biosciences (Parkville, VIC, Australia), non-essential amino acids from Gibco (Carlsbad, CA, USA) and pyruvate, uridine, HEPES, 2mercaptoethanol, protease inhibitor cocktail, STS, etoposide and MG132 were purchased from Sigma (St. Louis, MO, USA). Antibodies used for immunocytochemistry and Western blotting were mouse monoclonal cyt $c$ (clone 7H8.2C12 for Western blotting and clone 6H2.B4 for immunocytochemistry) and mouse monoclonal Bax (clone 6A7) from $B D$ Biosciences Pharmingen (San Jose, CA, USA); rat monoclonal Smacl DIABLO (clone 10G7, used for immunocytochemistry) from Apotech (Epalinges, Switzerland); rabbit polyclonal Smac/DIABLO (used for
Western blotting); mouse monoclonal pan-Actin from LabVision (Fremont, CA, USA); rabbit polyclonal Hsp60 was a gift from Professor $\mathrm{N}$ Hoogenraad; secondary antibodies conjugated to alkaline phosphatase for Western blotting detection were purchased from Chemicon (Temecula, CA, USA); rabbit anti-ubiquitin purchased from Sigma (St. Louis, MO, USA); goat anti-rabbit IgG, H\&L chain-specific peroxidase conjugate purchased from Calbiochem (from Merck Biosciences, Kilsyth, Vic, Australia); fluorescent secondary antibodies for immunocytochemistry conjugated to Alexa 488 or Alexa 568 and MTR were purchased from Molecular Probes (Eugene, OR, USA). Chemiluminescent detection using ECF substrate from GE Healthcare (Chalfont St. Giles, UK) or ECL detection using the Immun-Star HRP Substrate Kit from Bio-Rad (Hercules, CA, USA) was used for Western blotting detection. The Dead-End Fluorometric TUNEL System was purchased from Promega (Madison, WI, USA) and the caspase-3 activity kit purchased from Roche Applied Science (Mannheim, Germany).

\section{Cell culture}

Human osteosarcoma cells 143B TK- were cultured in RPMI 1640 medium supplemented with $10 \mathrm{mM}$ HEPES, $100 \mu \mathrm{M}$ penicillin/streptomycin and $10 \%$ foetal calf serum. Mouse embryonic NIH3T3 cells were cultured in DMEM medium supplemented as above. Cyt $c-1-$ cells (ATCC no. CRL-2613) and $\rho^{0} 206$ cells (derived from 143B TK- cells ${ }^{28}$ ) were cultured in DMEM supplemented with $10 \mathrm{mM}$ HEPES, $100 \mu \mathrm{M}$ penicillin/streptomycin, $10 \%$ foetal calf serum, $0.05 \mathrm{mM}$ 2-mercaptoethanol, $0.1 \mathrm{mM}$ nonessential amino acids, $0.11 \mathrm{mg} / \mathrm{ml}$ pyruvate and $0.05 \mathrm{mg} / \mathrm{ml}$ uridine. All cells were cultured in a humidified incubator at $37^{\circ} \mathrm{C}$ with $5 \% \mathrm{CO}_{2}$.

\section{Apoptotic inducers}

Cells were treated with STS or etoposide at the concentrations and times specified in individual figures.

\section{Cellular fractionation and Western blotting}

Fractionation of digitonin permeabilised cells was carried out according to Adrain et al. $^{29}$ to separate the mitochondrial and cytosolic proteins. Briefly, cells were collected, washed with PBS and resuspended in cytosolic lysis buffer $\left(250 \mathrm{mM}\right.$ sucrose, $70 \mathrm{mM} \mathrm{KCl}, 137 \mathrm{mM} \mathrm{NaCl}, 4.3 \mathrm{mM} \mathrm{NaPO}_{4}$, $1.4 \mathrm{mM} \mathrm{KH}_{2} \mathrm{PO}_{4}$, protease inhibitor cocktail) for $5 \mathrm{~min}$ on ice. Cells were centrifuged at $1000 \times g$ for 5 min and the supernatant was collected as the cytosolic fraction. The remaining pellet was resuspended in mitochondrial lysis buffer ( $50 \mathrm{mM}$ Tris- $\mathrm{HCl} \mathrm{pH} 7.4,150 \mathrm{mM} \mathrm{NaCl}, 2 \mathrm{mM}$ EDTA, $2 \mathrm{mM}$ EGTA, $0.2 \%$ Triton X-100, 0.3\% NP-40, protease inhibitor cocktail) for $5 \mathrm{~min}$ on ice. The suspension was centrifuged at $10000 \times g$ for $5 \mathrm{~min}$ and the supernatant collected as the mitochondrial fraction. Proteins from fractionated cells or unfractionated whole-cell lysates were resolved on $12 \%$ SDS-PAGE, transferred to PVDF membrane and probed with antibodies to cyt $c$ and Smac/DIABLO. Detection of ECF substrate and imaging was performed on a Storm Phosphoimager (GE Healthcare, Chalfont St. Giles, UK), while ECL substrate was exposed to Hyperfilm (GE Healthcare, Chalfont St. Giles, UK).

\section{Immunocytochemistry and confocal imaging}

Cells for immunostaining were seeded onto coverslips and incubated overnight to establish adherence. Following treatment, cells were incubated with $100 \mathrm{nM}$ of the mitochondrial reporter dye, MTR for 
$30 \mathrm{~min}$ at $37^{\circ} \mathrm{C}$ to label mitochondria. Cells were then fixed with $3.5 \%$ paraformaldehyde for $10 \mathrm{~min}$ at $37^{\circ} \mathrm{C}$ and permeabilised with $0.1 \%$ Triton $\mathrm{X}-100$ at room temperature for $10 \mathrm{~min}$. In some experiments, cells were alternatively permeabilised with CHAPS buffer $(150 \mathrm{mM} \mathrm{NaCl}, 10 \mathrm{mM}$ HEPES, $1.0 \%$ CHAPS) after fixation. Cells were immunostained at $4{ }^{\circ} \mathrm{C}$ overnight with antibodies to cyt $c$, Smac/DIABLO and Bax. Cells were imaged with an Olympus Fluoview 500 inverted confocal laser scanning microscope equipped with an Argon/HeNe laser light source. Images were collected using a 60x/1.2 water immersion lens. In dual-channel imaging, photomultiplier sensitivities and offsets were set to a level at which bleed through effects from one channel to another were negligible. Scale bars on images were set at $50 \mu \mathrm{m}$.

\section{TUNEL analysis for DNA degradation}

Briefly, cells were collected, fixed in $1 \%$ paraformaldehyde for 20 min on ice, and then permeabilised in $70 \%$ ethanol overnight before incubation with FITC-conjugated anti-Tdt antibody for $1 \mathrm{~h}$ at $37^{\circ} \mathrm{C}$ Data collection were carried out using the FACSCalibur flow cytometer (BD Biosciences, San Jose, CA, USA) using an excitation wavelength of $488 \mathrm{~nm}$ and analysis on 10000 events per sample performed using CellQuest software.

\section{Analysis of active caspase-3}

The presence of active caspase- 3 was determined on a fluorescence plate reader (Flurostar Optima from BMG Labtech, Offenburg, Germany); excitation $405 \mathrm{~nm}$, emission $510 \mathrm{~nm}$. Cell lysates were incubated on a plate coated with antibody to active caspase-3. After removal of the lysate, the caspase- 3 substrate DEVD-AFC was added and incubated at $37^{\circ} \mathrm{C}$ for $2 \mathrm{~h}$ before analysis. Fluorescence values obtained from independent experiments were expressed as relative fluorescence units by comparison with 143B TK - cells treated with STS (this consistently provided the highest reading), which was taken to be 100 units.

\section{Acknowledgements}

The excellent technical assistance of Susan Ekkel is gratefully acknowledged. We thank Professor N Hoogenraad (La Trobe University) for his gift of antibodies specific for Hsp60, and Dr. R Baker (Australian National University) for helpful advice on proteasome inhibitors and ubiquitinylation. This work was supported by the Australian Research Council.

\section{References}

1. Desagher S and Martinou JC (2000) Mitochondria as the central control point of apoptosis. Trends Cell Biol. 10: 369-377

2. Wei MC, Zong WX, Cheng EH, Lindsten T, Panoutsakopoulou V, Ross AJ, Roth KA, MacGregor GR, Thompson CB and Korsmeyer SJ (2001) Proapoptotic $\mathrm{BAX}$ and $\mathrm{BAK}$ : a requisite gateway to mitochondrial dysfunction and death. Science 292: 727-730

3. Verhagen AM, Ekert PG, Pakusch M, Silke J, Connolly LM, Reid GE, Moritz RL, Simpson RJ and Vaux DL (2000) Identification of DIABLO, a mammalian protein that promotes apoptosis by binding to and antagonizing IAP proteins. Cell 102: 43-53

4. Du C, Fang M, Li Y, Li L and Wang X (2000) Smac, a mitochondrial protein that promotes cytochrome $c$-dependent caspase activation by eliminating IAP inhibition. Cell 102: 33-42

5. Susin SA, Lorenzo HK, Zamzami N, Marzo I, Snow BE, Brothers GM, Mangion J, Jacotot E, Costantini P, Loeffler M, Larochette N, Goodlett DR, Aebersold R,
Siderovski DP, Penninger JM and Kroemer G (1999) Molecular characterization of mitochondrial apoptosis-inducing factor. Nature 397 : 441-446

6. Li K, Li Y, Shelton JM, Richardson JA, Spencer E, Chen ZJ, Wang X and Williams RS (2000) Cytochrome $c$ deficiency causes embryonic lethality and attenuates stress-induced apoptosis. Cell 101: 389-399

7. Bertrand R, Solary E, O'Connor P, Kohn KW and Pommier Y (1994) Induction of a common pathway of apoptosis by staurosporine. Exp. Cell Res. 211: 314-321

8. Froelich-Ammon SJ and Osheroff $N$ (1995) Topoisomerase poisons: harnessing the dark side of enzyme mechanism. J. Biol. Chem. 270: 21429-21432

9. Hengartner MO (2000) The biochemistry of apoptosis. Nature 407: 770-776

10. Takasawa $R$ and Tanuma $S$ (2003) Sustained release of Smac/DIABLO from mitochondria commits to undergo UVB-induced apoptosis. Apoptosis 8: 291-299

11. Karpinich NO, Tafani M, Rothman RJ, Russo MA and Farber JL (2002) The course of etoposide-induced apoptosis from damage to DNA and p53 activation to mitochondrial release of cytochrome c. J. Biol. Chem. 277: 16547-16552

12. Jiang S, Cai J, Wallace DC and Jones DP (1999) Cytochrome c-mediated apoptosis in cells lacking mitochondrial DNA. Signaling pathway involving release and caspase 3 activation is conserved. J. Biol. Chem. 274: 29905-29911

13. MacFarlane M, Merrison W, Bratton SB and Cohen GM (2002) Proteasomemediated degradation of Smac during apoptosis: XIAP promotes Smac ubiquitination in vitro. J. Biol. Chem. 277: 36611-36616

14. Antonsson B, Montessuit S, Lauper S, Eskes R and Martinou JC (2000) Bax oligomerization is required for channel-forming activity in liposomes and to trigger cytochrome $c$ release from mitochondria. Biochem. J. 345: $271-278$

15. Kuwana T, Mackey MR, Perkins G, Ellisman MH, Latterich M, Schneiter R, Green DR and Newmeyer DD (2002) Bid, Bax, and lipids cooperate to form supramolecular openings in the outer mitochondrial membrane. Cell 111: 331-342

16. Rehm M, Dussmann $\mathrm{H}$ and Prehn $\mathrm{JH}$ (2003) Real-time single cell analysis of Smac/DIABLO release during apoptosis. J. Cell Biol. 162: 1031-1043

17. Springs SL, Diavolitsis VM, Goodhouse J and McLendon GL (2002) The kinetics of translocation of Smac/DIABLO from the mitochondria to the cytosol in HeLa cells. J. Biol. Chem. 277: 45715-45718

18. Chai J, Du C, Wu JW, Kyin S, Wang $X$ and Shi $Y$ (2000) Structural and biochemical basis of apoptotic activation by Smac/DIABLO. Nature 406: 855-862

19. Wang JL, Liu D, Zhang ZJ, Shan S, Han X, Srinivasula SM, Croce CM, Alnemri ES and Huang Z (2000) Structure-based discovery of an organic compound that binds Bcl-2 protein and induces apoptosis of tumor cells. Proc. Natl Acad. Sci. USA 97: 7124-7129

20. An J, Chen $Y$ and Huang $Z$ (2004) Critical upstream signals of cytochrome $c$ release induced by a novel bcl-2 inhibitor. J. Biol. Chem. 279: 19133-19140

21. Scorrano L, Ashiya M, Buttle K, Weiler S, Oakes SA, Mannella CA and Korsmeyer SJ (2002) A distinct pathway remodels mitochondrial cristae and mobilizes cytochrome $c$ during apoptosis. Dev. Cell 2: 55-67

22. Boehning D, Patterson RL, Sedaghat L, Glebova NO, Kurosaki T and Snyder SH (2003) Cytochrome $c$ binds to inositol $(1,4,5)$ trisphosphate receptors, amplifying calcium-dependent apoptosis. Nat. Cell Biol. 5: 1051-1061

23. Orrenius S, Zhivotovsky B and Nicotera P (2003) Regulation of cell death: the calcium-apoptosis link. Nat. Rev. Mol. Cell Biol. 4: 552-565

24. Arnoult D, Gaume B, Karbowski M, Sharpe JC, Cecconi F and Youle RJ (2003) Mitochondrial release of AIF and EndoG requires caspase activation downstream of Bax/Bak-mediated permeabilization. EMBO J. 22: 4385-4399

25. Schmitt E, Parcellier A, Ghiringhelli F, Casares N, Gurbuxani S, Droin N, Hamai A, Pequignot M, Hammann A, Moutet M, Fromentin A, Kroemer G, Solary E and Garrido $C$ (2004) Increased immunogenicity of colon cancer cells by selective depletion of cytochrome c. Cancer Res. 64: 2705-2711

26. Waterhouse NJ, Goldstein JC, von Ahsen O, Schuler M, Newmeyer DD and Green DR (2001) Cytochrome $c$ maintains mitochondrial transmembrane potential and ATP generation after outer mitochondrial 
membrane permeabilization during the apoptotic process. J. Cell Biol. 153: 319-328

27. Jiang S, Cai J, Wallace DC and Jones DP (1999) Cytochrome c-mediated apoptosis in cells lacking mitochondrial DNA. Signaling pathway involving release and caspase 3 activation is conserved. J. Biol. Chem. 274: 29905-29911
28. King MP and Attardi G (1989) Human cells lacking mtDNA: repopulation with exogenous mitochondria by complementation. Science 246 : 500-503

29. Adrain C, Creagh EM and Martin SJ (2001) Apoptosis-associated release of Smac/DIABLO from mitochondria requires active caspases and is blocked by Bcl-2. EMBO J. 20: 6627-6636 